\title{
Percepção de fonoaudiólogos sobre a atuação na área de cuidados paliativos em um hospital público de Santa Catarina
}

\section{Speech-language pathologists' perception on their performance in the} palliative care area at a public hospital in Santa Catarina state, Brazil

\author{
Brenda Nathalye Nunes Mendes ${ }^{1}$ (D), Mara Keli Christmann ${ }^{1,2}$ (D), Jelson Budal Schmidt ${ }^{3}$ (D), \\ Eduarda Silveira de $\mathrm{Abreu}^{2}$ (D)
}

\begin{abstract}
RESUMO
Objetivo: identificar a percepção de fonoaudiólogos sobre a própria atuação em cuidados paliativos em um hospital público de Santa Catarina. Método: trata-se de um estudo qualitativo, descritivo, realizado com cinco fonoaudiólogos atuantes em um hospital público de Santa Catarina. Os dados foram coletados mediante entrevista semiestruturada e submetidos à análise de conteúdo. Resultados: após análise do conteúdo do discurso dos fonoaudiólogos participantes, surgiram as seguintes categorias: conceito e entendimento sobre cuidados paliativos e atuação do fonoaudiólogo; reconhecimento da Fonoaudiologia na atuação multiprofissional; relação interpessoal com o paciente e a família e dificuldades na prática de cuidados paliativos. Conclusão: a percepção dos fonoaudiólogos revela o conceito de que os cuidados paliativos são prestados a pacientes que não possuem mais possibilidade de cura, mas para os quais há necessidade de tratar comorbidades e proporcionar melhor qualidade de vida. Evidenciaram-se durante as entrevistas: a percepção da atuação fonoaudiológica na área da disfagia, o reconhecimento dos demais profissionais de que a atuação fonoaudiológica tem se ampliado e de que o cuidado deve se estender a toda a família.
\end{abstract}

Palavras-chave: Cuidados paliativos; Fonoaudiologia; Equipe de assistência ao paciente; Percepção; Hospitalar

\begin{abstract}
Purpose: To identify speech-language pathologists' perception on their performance in palliative care at a public hospital in Santa Catarina State, Brazil. Methods: It is a descriptive, qualitative study, conducted by 5 speech-language pathologists at a public hospital in Santa Catarina State. Data were collected by means of a semi-structured interview and submitted to content analysis. Results: After the content analysis of the participating speech-language pathologists, the following post-defined categories emerged: concept and understanding of palliative care and speech-language pathologists' performance; the importance of the speechlanguage pathologists in multidisciplinary teams; interpersonal relationship with patients and their families, and constraints in the practice of palliative care. Conclusion: According to speech-language pathologists' perception, palliative care is delivered to patients without any possibility of cure, but it is necessary to treat their comorbidities, and provide them with better quality of life; the perception of the speech-language pathology performance in the field of dysphagia stood out; report of other professionals acknowledging speech-language pathologists' performance in the area of dysphagia has been expanded; and such care should be extended to the whole family.
\end{abstract}

Keywords: Palliative care; Speech-Language Pathology; Patient support team; Perception; Hospital

Trabalho realizado na Associação Educacional Luterana BOM JESUS - IELUSC - Joinville (SC), Brasil.

${ }^{1}$ Curso de Fonoaudiologia, Associação Educacional Luterana BOM JESUS - IELUSC - Joinville (SC), Brasil.

${ }^{2}$ Universidade do Vale do Itajaí - UNIVALI - Itajaí (SC), Brasil.

${ }^{3}$ Curso de Fonoaudiologia e Educação Física, Associação Educacional Luterana BOM JESUS - IELUSC - Joinville (SC), Brasil.

Conflito de interesses: Não.

Contribuição dos autores: BNNM participou da idealização do estudo, coleta, análise e interpretação dos dados e redação do artigo; MKC participou da idealização do estudo, coleta, análise e interpretação dos dados e redação do artigo; JBS participou da idealização do estudo, coleta, análise e interpretação dos dados e redação do artigo; ESA participou da revisão do artigo e edição para a revista.

Financiamento: Nada a declarar.

Autor correspondente: Brenda Nathalye Nunes Mendes. E-mail: brenda-nathalye1@hotmail.com

Recebido: Setembro 06, 2021; Aceito: Novembro 01, 2021 


\section{INTRODUÇÃO}

A Organização Mundial de Saúde (OMS) definiu o termo "cuidados paliativos" em 1990, que foi revisado em 2002, como sendo todo cuidado prestado aos pacientes que possuem alguma doença que ameace a vida. Esse cuidado é dispensado por uma equipe multiprofissional, com o objetivo de promover melhor qualidade de vida e alívio dos sofrimentos ${ }^{(1)}$.

Atualmente, existem objetivos gerais que regem a atuação dos profissionais nessa área, como controle da dor e outros sintomas, tanto físicos como emocionais, priorizar o cuidado e não a cura, acreditar que a morte é um processo natural, sem antecipar e nem prolongar esse fato, proporcionar autonomia ao paciente, acolher a família durante todo o processo da doença e, após, na fase do luto, e sempre privilegiar as decisões compartilhadas, da maneira mais ética possível ${ }^{(1)}$.

Para dar conta de abranger o controle da sintomatologia que surge em grande parte das doenças em estágio avançado, recentemente houve a inserção do fonoaudiólogo como parte da equipe. Esse profissional auxilia na comunicação ${ }^{(2)}$ e deglutição do paciente, proporcionando melhor qualidade de vida, por meio de estratégias de reabilitação e monitoramento dessas funções ${ }^{(3)}$.

O fonoaudiólogo hospitalar, em geral, atua na prevenção e diminuição das sequelas causadas à linguagem, deglutição e motricidade orofacial do paciente, possibilitando uma recuperação eficiente e, assim, aumentando as possibilidades de reintegração do paciente à sociedade após alta hospitalar ${ }^{(4)}$. No caso de atendimento, especificamente na área de cuidados paliativos hospitalares, o objetivo costuma ser o de manter deglutição prazerosa e segura para o enfermo pelo máximo de tempo possível, bem como a comunicação eficiente ${ }^{(2)}$, para que seja possível a ele expressar suas decisões no tratamento e para que exista o contato entre familiares, equipe e paciente, gerando melhor qualidade de vida e um atendimento humanizado ao final da vida, sem esquecer as limitações impostas pela própria doença de base. Salienta-se que, ao atender um enfermo que necessita de cuidados paliativos, a atuação profissional não é apenas ao paciente, mas se estende a sua família ${ }^{(3)}$.

Para o profissional que atende na área de cuidados paliativos, a perspectiva é diferente, o foco não é no diagnóstico, pois esses cuidados não visam medidas de cura da doença de base, mas o alívio do sofrimento e a melhora da qualidade de vida, o que varia conforme cada caso ${ }^{(5)}$. Esse aspecto pode representar um sofrimento emocional não só para o paciente, mas para os profissionais que atuam junto a ele, de modo que, possivelmente, não sejam todos os profissionais, de cada categoria, que desejam trabalhar no âmbito de cuidados paliativos.

A percepção dos profissionais atuantes na equipe multiprofissional reflete nas atividades praticadas diariamente no hospital, pois é por meio da consciência em relação às necessidades sociais locais e do conhecimento científico, que haverá o aperfeiçoamento das atuações ${ }^{(6)}$. Por esse motivo faz-se importante compreender a visão dos profissionais envolvidos nessa equipe.

Tendo em vista que a literatura é escassa sobre a percepção dos fonoaudiólogos em relação à atuação em cuidados paliativos, há poucos materiais de consulta específicos para os fonoaudiólogos que almejam trabalhar nessa área. Os estudos recentemente publicados, relacionados à Fonoaudiologia e cuidados paliativos, evidenciam a importância da atuação nesta área ${ }^{(7,8)}$, porém, pouco se sabe sobre a percepção dos profissionais sobre a atual prática. A proposta de discussão do objeto deste estudo contribuirá para a ciência fonoaudiológica e atuação na área hospitalar.

Sendo assim, objetivou-se, com este estudo, identificar a percepção de fonoaudiólogos sobre a própria atuação em cuidados paliativos em um hospital público de Santa Catarina.

\section{MÉTODOS}

Trata-se de um estudo do tipo exploratório, descritivo, transversal, de caráter qualitativo.

O presente projeto de pesquisa foi aprovado pelo Comitê de Ética em Pesquisa (CEP) da Associação Educacional Luterana BOM JESUS/IELUSC (parecer $\mathrm{n}^{\circ}$ 4.055.458).

Os critérios de inclusão para os participantes da pesquisa foram: ser fonoaudiólogo e possuir experiência de trabalho hospitalar em cuidados paliativos. Os critérios de exclusão foram: fonoaudiólogos que atuassem há menos de seis meses na área hospitalar, por não possuírem experiência profissional necessária para os devidos relatos. A amostra foi selecionada por processo de amostragem não probabilística de conveniência, resultando em seis fonoaudiólogos atuantes em um hospital do norte de Santa Catarina. Entretanto, obteve-se resposta de cinco deles. O hospital é público e atende demandas de alta complexidade da região.

Devido à pandemia da Covid-19 e às medidas adotadas para sua contenção, optou-se pela realização da coleta de dados de forma on-line, utilizando-se a plataforma Google Meet. A coleta ocorreu durante o mês de agosto de 2020, em horários previamente combinados entre a pesquisadora e os participantes, individualmente, por meio de entrevista semiestruturada, gravada com autorização dos participantes. O questionário semiestruturado (Anexo 1) utilizado para a entrevista foi baseado na literatura ${ }^{(9)}$ e na experiência dos pesquisadores, uma vez que não foram encontrados estudos qualitativos abordando os mesmos aspectos do presente trabalho.

As entrevistas foram gravadas e, posteriormente, transcritas de modo integral. Em seguida, foram agrupados os dados obtidos por semelhança de assuntos, verificando os aspectos que se destacaram, para definição do conteúdo abordado pelos participantes, ou seja, as percepções dos mesmos. Todos os indivíduos envolvidos na pesquisa assinaram o Termo de Consentimento Livre e Esclarecido.

As etapas para realização do levantamento de dados seguiram a proposta de Minayo ${ }^{(10)}$, conforme segue: coleta dos dados, leitura do conteúdo, separação e definição das categorias. Já os dados foram analisados segundo a proposta de $\operatorname{Bardin}^{(11)}$, com análise de conteúdo.

Com o intuito de garantir o anonimato, os participantes da pesquisa foram identificados de acordo com a profissão, seguida de um número, como, por exemplo: Fonoaudiólogo 1, Fonoaudiólogo 2 e assim por diante, expressas pelas siglas F1, F2 e assim, sucessivamente.

\section{RESULTADOS}

Foram convidados a participar 6 profissionais que atuavam no hospital escolhido para a realização da pesquisa, obtendo-se resposta de 5 deles. Considerando-se tratar de uma pesquisa 
qualitativa, optou-se por apresentar a caracterização prévia dos participantes:

Identificou-se que houve maior ocorrência do gênero feminino, representando $4(80 \%)$ dos 5 indivíduos. O tempo de formação oscilou entre 10 e 32 anos, existindo uma concentração entre 10 e 20 anos. Já com relação ao tempo de atuação, houve uma variação entre 2 e 20 anos, com maior evidência entre 6 e 9 anos, conforme descrito na Tabela 1:

Por meio da análise minuciosa das entrevistas dos fonoaudiólogos, surgiram as seguintes categorias definidas $a$ posteriori: conceito e entendimento sobre cuidados paliativos e atuação do fonoaudiólogo; reconhecimento da Fonoaudiologia na atuação multiprofissional; relação interpessoal com o paciente e a família e dificuldades na prática de cuidados paliativos.

\section{Conceito e entendimento sobre cuidados paliativos e atuação do fonoaudiólogo}

Esse tema surgiu a partir do questionamento a respeito da percepção sobre cuidados paliativos. A maioria dos participantes referiu que os cuidados paliativos são prestados a pacientes que não possuem mais possibilidade de cura, mas para os quais é possível tratar comorbidades e, principalmente, proporcionar melhor qualidade de vida, conforme se observou nas falas a seguir:

F1: "É um cuidado com o paciente, onde os tratamentos já não terão um poder curativo pra ele, não vai reverter ele da doença, da possibilidade iminente de morte. Visa dar um conforto, uma forma digna dele viver a terminalidade dos dias que ainda o restam e que ele não sofra [...] que ele não tenha dor, não tenha sofrimento físico agonizante." F3 concordou com o entendimento de F1, quando este abordou, em seu relato, a qualidade de vida em diversos aspectos, com a fala: "[...] religioso e de saúde. Significa não curar, mas pode ser amenizado dor e sintomas." (F3).

Já o participante $\mathrm{F} 4$, além de mencionar a melhor qualidade de vida a ser proporcionada, citou, também, um ponto de grande relevância em cuidados paliativos, a finitude da vida: “[...] se possível, que o paciente esteja ciente da finitude, porque é só na ciência de que está chegando ao fim, que você pode fazer o que talvez não tinha feito."

De acordo com F3, o conceito de cuidados paliativos é algo pequeno, comparado à aplicação no dia a dia, sendo comum não ser aplicado em sua totalidade nos atendimentos, conforme verificado no relato a seguir: "O conceito do que é paliativo eu vejo que as pessoas têm, que é tratar na íntegra o paciente, então, com tudo que você tem de recurso, mas no dia a dia,

Tabela 1. Caracterização dos participantes

\begin{tabular}{cccc}
\hline Entrevistados & Gênero & $\begin{array}{c}\text { Tempo de } \\
\text { formação }\end{array}$ & $\begin{array}{c}\text { Tempo de } \\
\text { atuação } \\
\text { na área } \\
\text { hospitalar }\end{array}$ \\
\hline F1 & F & 32 anos & 9 anos \\
F2 & M & 12 anos & 2 anos \\
F3 & F & 20 anos & 20 anos \\
F4 & F & 10 anos & 7 anos \\
F5 & F & 23 anos & 6 anos \\
\hline
\end{tabular}

Fonte: dados da pesquisa, 2020

Legenda: $F 1,2,3,4$ = fonoaudiólogo 1, 2, 3, 4; F = feminino; $M=$ masculino quando se fala que é um cuidado paliativo, as pessoas só pensam que não irá ser mais feito nada pelo paciente e então se perde o conceito real do paliativo".

F3 também salientou: "O papel do fonoaudiólogo é comunicação e disfagia, não podemos pensar só na deglutição. Fazer com que o paciente consiga se comunicar e comer, mesmo que não seja para suprir suas necessidades nutricionais, mas que seja prazeroso, sempre buscando pela qualidade de vida dele."

Já F4 e F1, discorreram mais a fundo sobre o trabalho específico na disfagia: "[...] às vezes, a gente indica ou não uma via alternativa de alimentação, depende muito do prognóstico, qual é a patologia. Às vezes só uma adaptação de consistência ou liberamos umas coisas, mesmo sabendo que há risco de aspirar, só para prazer alimentar mesmo.” (F4). "[...] quando o paciente deseja muito experimentar algo que ele gostava, podemos conseguir um meio de promover essa degustação do sabor, sem que lhe cause um grande mal." (F1).

O participante F3 complementou e explicou sobre a colocação de F4, ao citar que: “[...] há casos que o paciente aspira e a família continua optando por dar comida. Ele pode correr o risco de ter uma pneumonia, mas não muda o quadro clínico, porque já não há mais perspectiva de melhora, então, é mantido o único prazer que ele tem".

O participante F1 mencionou esta prática vivenciada: "[...] teve uma paciente com esclerose lateral amiotrófica que não conseguia se comunicar, e ela queria falar; então, estabelecemos uma linguagem alternativa pra ela dizer o que estava sentindo e pensando."

Nesta categoria, pôde-se identificar que o conceito de cuidados paliativos é aplicado de forma parcial, porém, há o entendimento dos participantes do que sejam esses cuidados. Com relação à atuação do fonoaudiólogo, pôde-se notar que, em todos os relatos, sobressaiu-se o atendimento na área da disfagia.

\section{Reconhecimento da fonoaudiologia na atuação multiprofissional}

Sendo assim, o reconhecimento não só na área de cuidados paliativos, mas de todas as atividades oferecidas dentro do hospital, também é um entendimento em ascensão na vida dos profissionais da Fonoaudiologia, como observado nas fala de F5 e F1, "[...] atualmente, eu vejo que a equipe médica tem muito respeito pelo meu trabalho, respeitam minhas condutas, solicitam muito minha avaliação e fazem esse link, só liberam se a fonoaudióloga liberar. Melhorou muito!" (F5). "Hoje em dia, a gente é muito reconhecido. A princípio, foi um trabalho muito de formiguinha e a neurologia que valorizava mais, mas, ao longo dos anos, as outras clínicas também foram respeitando $[\ldots] "$ ".. (F1).

Já F3, além de discorrer sobre o progresso do reconhecimento, citou a dificuldade dos profissionais e pacientes em compreenderem sobre todas as áreas de atuação fonoaudiológica dentro de um hospital: "[...] muitos não sabem o que a gente faz, até mesmo os profissionais da saúde, técnicos, radiologistas... inclusive paciente, que acha que só atendemos problemas na fala".

F4 também expressou, em sua fala, o desejo de disseminar o conhecimento sobre os cuidados paliativos no hospital em questão:

Temos um caminho bem grande a trilhar com cuidados paliativos, gostaríamos de ter uma equipe só de cuidados 
paliativos, ou pelo menos, ter um grupo para dar orientações e palestras, que nem as palestras já realizadas sobre disfagia, para os residentes e copeiras.

O participante $\mathrm{F} 2$ referiu outro ponto relevante, a respeito da ampla atenção dada à disfagia, gerando, assim, um reconhecimento maior nessa área: "[...] quando reabilita um paciente e $o$ paciente volta a se alimentar, tira a sonda, é decanulado, a gente percebe, sim, o reconhecimento desses profissionais", sendo complementado pela fala de F4, ao citar que: "É mais quando a gente consegue progredir no sentido de melhorar um nível. Se eu libero uma comida, uma consistência, tiro uma sonda, tiro uma gastrostomia, aí eles reconhecem, mas é no sentido de progressão."

\section{Relação interpessoal com o paciente e a família e dificuldades na prática de cuidados paliativos}

Por meio das falas, evidenciou-se que a família constituise como um dos grandes pilares para o cuidado efetivo, assim como citado na fala de F4, quando expôs que: "Às vezes a gente é só uma voz, uma ponte entre paciente, familiar e médico ou responsável da equipe. Quando vamos com um pouquinho mais de tempo, paciência, conseguimos resgatar coisas da família e do paciente que são importantes e aí passa para a equipe ou faz uma ponte entre o próprio familiar e paciente."

A fala de F5 demonstrou essa atenção evidenciada na literatura: "Eu acho que além do paciente paliativo, temos que envolver a familia; também deve ser tratada e merece esse cuidado"

Ao discorrer sobre a família, surgiu, na fala de F4, um dos impasses na vivência em cuidados paliativos, ao relatar que: "Tem casos que a família quer esconder diagnóstico e isso não é bacana, porque o paciente tem o direito, por lei, de saber e isso pode causar uma transformação nele [...] Então você precisa falar com os familiares sobre aceitar o fim, que esse é o caminho da vida", estando de acordo com Silva e Sudigursky ${ }^{(12)}$, ao afirmarem que existem princípios éticos que regem os cuidados paliativos, sendo um deles a veracidade, isto é, dizer sempre a verdade à família e ao paciente, inclusive com relação à finitude da vida, com o objetivo de proporcionar um processo de morte menos doloroso e de paz.

Focalizando nesses dilemas, surgiu a reflexão sobre os casos de caquexia, nos quais o paciente tem uma redução extrema de peso e as deficiências nutricionais não conseguem mais ser corrigidas, gerando um grande sofrimento por parte da família, principalmente por associarem à ideia de que o paciente irá falecer em razão de não se alimentar, sendo necessária a efetiva comunicação e o suporte psicossocial, como exposto no relato de F3:

O dilema da família querer que o paciente coma e ele não só não precisa mais, como não consegue, porque quando o paciente ainda tem consciência, o cognitivo preservado e tem o desejo, ai fazemos por isso, mas tem um momento nos internados que eles não têm mais essa consciência, esse é o maior problema que eu vejo, da família achar que ele tem que comer, quando não precisa e oferta comida pro paciente dormindo e prejudica, isso acontece com muita frequência.

Além da dificuldade de a família compreender sobre as condutas, o participante F4 trouxe, em sua fala, a dificuldade dos próprios profissionais nessa concepção: “[...] não só em questão a familia, mas aos profissionais também, e até meu mesmo de entender no começo, não vão passar uma sonda, mas por quê? Talvez vivesse mais uma semana se passasse a sonda. É mais no sentido de entender a conduta."

\section{DISCUSSÃO}

Os resultados obtidos de acordo com as falas na categoria "conceito e entendimento sobre cuidados paliativos e atuação do fonoaudiólogo" estão de acordo com um autor ${ }^{(13: 17)}$ que retrata o entendimento da morte como um processo natural do ciclo de vida, torna-o menos doloroso e permite que o paciente possa ter autonomia para viver do jeito que deseja ou, simplesmente, optar por não sofrer mais com as medidas invasivas.

A maioria dos profissionais entrevistados possuía muitos anos de trabalho em ambiente hospitalar e, com isso, provavelmente acompanharam a evolução da modificação da filosofia sobre os cuidados paliativos, tendo, possivelmente, ressignificado alguns entendimentos ao longo dos anos, substituindo, por exemplo, a visão de "não tem mais o que fazer" pela visão atual de que "não foca mais na impossibilidade de cura, mas na possibilidade de proporcionar alívio de sintomas da doença e, assim, qualidade de vida do paciente" (14:7). Segundo a literatura ${ }^{(15)}$,só se adquire a habilidade em trabalhar com o cuidado humanizado quando este é colocado em prática, desenvolvendo-se, assim, maior competência e aperfeiçoamento profissional.

Em relação aos relatos sobre os conceitos e a compreensão dos participantes, prosseguiu-se para a atuação do fonoaudiólogo em cuidados paliativos, sendo seu objetivo proporcionar melhor comunicação, tornando possível ao paciente refletir sobre seus desejos quando estiver consciente e possibilitar a alimentação mais segura e prazerosa possível ${ }^{(2)}$. Uma publicação ${ }^{(16)}$ relata que, ao optar por uma via alternativa de alimentação, normalmente a oferta mínima por via oral é priorizada, para satisfazer o desejo do paciente ${ }^{(5)}$.

Outra publicação(17) defende que a grande importância atribuída à disfagia ocorre devido à atuação tradicional do fonoaudiólogo em priorizar a redução de riscos relacionados a essa dificuldade, com o intuito de prevenir o acarretamento de mais complicações, como pneumonia ou insuficiência respiratória. Entretanto, ao focar nos cuidados paliativos, existem casos em que o paciente opta por continuar com a alimentação por via oral, apesar dos riscos. Nesses casos, sabe-se que deve ser realizada uma discussão com toda a equipe multiprofissional para que a decisão da manutenção da via oral seja compartilhada, mas centrada no paciente ${ }^{(5,18)}$. É de competência do fonoaudiólogo informar sobre os riscos, respeitar a decisão do paciente e minimizar a possibilidade de broncoaspiração, por meio de manobras, orientações e adaptações.

Já nos casos em que a doença de base afetou a comunicação efetiva do paciente, impossibilitando-o de expressar seus desejos, o fonoaudiólogo pode buscar a utilização de meios alternativos de comunicação, como gestos, escritas, desenhos e pranchas de comunicação( ${ }^{(2)}$. Essa atuação específica em relação à comunicação mostrou-se muito pouco nas falas dos entrevistados, o que pode ser explicado devido ao fato de hospital em questão não possuir uma equipe específica para cuidados paliativos, não existindo a centralização de profissionais direcionados apenas à atenção de pacientes em cuidados paliativos. Dessa forma, os fonoaudiólogos atendem os setores para os quais são designados, bem como os pacientes que estão em cuidados 
paliativos dentro desses setores. Outro fator para a maior atenção conferida para a questão da disfagia é o fato de que, em geral, há uma demanda cada vez mais crescente nos hospitais pelos atendimentos fonoaudiológicos e o número de profissionais ainda é reduzido, possivelmente limitando o tempo hábil para dispender com todos os pacientes em cuidados paliativos as questões relativas à comunicação. Além disso, ressalva-se o próprio histórico da área de Fonoaudiologia hospitalar, em que a atuação se iniciou, primeiramente, aos pacientes com disfagia, devido às doenças neurológicas, e, posteriormente, englobou os casos de linguagem ${ }^{(12)}$.

A decisão compartilhada de qual ação perante o paciente é um ponto chave entre os profissionais da equipe multiprofissional, já que os cuidados devem envolver os sintomas físicos, psicológicos, espirituais e sociais e, assim, necessitam da atuação dos mais diversos profissionais, sendo de competência do fonoaudiólogo e dos outros profissionais da saúde disseminar essa ideia e buscar o reconhecimento ${ }^{(18)}$.

O papel do fonoaudiólogo na equipe multiprofissional em cuidados paliativos é relativamente novo, surgindo com o conceito de que, para se obter um cuidado humanizado, é necessária a comunicação do paciente e a segurança durante a alimentação ${ }^{(19)}$. Sendo assim, percebe-se que o reconhecimento da atuação do fonoaudiólogo hospitalar é crescente entre os profissionais, o que ficou evidente nas falas dos entrevistados.

Salienta-se que o trecho em que F4 cita "tiro uma sonda, tiro uma gastrostomia", ilustrou a importância que a equipe multiprofissional desse hospital direciona ao fonoaudiólogo, no que se refere à responsabilidade quanto às condições do paciente alimentados por via oral exclusiva. Certamente F4 se referiu ao fato de sugerir a retirada da via alternativa, uma vez que é a equipe médica que prescreve tal retirada.

Faz-se necessário, ainda, divulgar e informar os pacientes, familiares e profissionais da saúde, de um modo geral, sobre as possibilidade de atuação do fonoaudiólogo na área de cuidados paliativos, para que tenham a oportunidade de solicitar o atendimento fonoaudiológico quando necessário e, assim, não considerem o trabalho desse profissional exclusivamente ao atendimento clínico em consultório ${ }^{(20)}$. Sabe-se que, na área de cuidados paliativos, as medidas dispensadas ao paciente possuem caráter readaptativo e não curativo ${ }^{(3)}$, o que não tende a ocasionar em melhora da doença. Sendo assim, compreendese que o reconhecimento da equipe multiprofissional perante os fonoaudiólogos, nessa, área torna-se ainda mais complexo.

A família constitui-se como um dos grandes pilares para o cuidado efetivo. Construir uma boa relação com a família possibilita a melhor comunicação da equipe com o paciente, além de permitir que ela manifeste opinião sobre os cuidados prestados e, portanto, trabalhe em conjunto com a equipe multiprofissional, em prol do cuidado mais humanizado possível ${ }^{(21)}$. O "cuidar", neste caso, não se refere somente ao enfermo, mas à família, buscando deixá-los sempre informados, orientados e seguros diante das intervenções realizadas ${ }^{(21,22)}$.

Um aspecto que pode dificultar a atuação em cuidados paliativos junto à família é o fato de que, durante a própria graduação dos profissionais, não são abordadas questões sobre o processo de morrer, o que dificulta a inserção e o "saber lidar" desses profissionais na equipe de cuidados paliativos ${ }^{(18)}$.

Aliando-se ao fato da real complexidade desse assunto, sugerese que as decisões e condutas gerais sejam tomadas mediante discussões do caso em conjunto com todos os participantes da equipe, mantendo sempre a família e o paciente informados e com possibilidade de participação no processo ${ }^{(23)}$.

O presente estudo se destaca por ser o primeiro, até a data de elaboração, a evidenciar a percepção dos fonoaudiólogos na área de cuidados paliativos no Brasil e demonstrar uma realidade local. Entretanto, aponta-se como limitação da pesquisa o fato de ter sido aplicada em apenas uma instituição. Assim, sugere-se que, em estudos futuros, seja ampliado o número de participantes, para verificar e confirmar os pontos de vista e conhecer as realidades distintas vivenciadas pelos fonoaudiólogos que atuam na área de cuidados paliativos.

Os resultados obtidos contribuem com a ciência na área da Fonoaudiologia, expondo uma visão sobre a realidade dos cuidados paliativos sob a ótica do profissional fonoaudiólogo, tanto para estudantes, como fonoaudiólogos e profissionais de diferentes áreas da saúde.

\section{CONCLUSÃO}

A percepção dos fonoaudiólogos revela o conceito de que os cuidados paliativos são prestados a pacientes que não possuem mais possibilidade de cura, mas para os quais há necessidade de tratar comorbidades e proporcionar melhor qualidade de vida. Evidenciaram-se durante as entrevistas: a atuação fonoaudiológica na área da disfagia; o reconhecimento dos demais profissionais de que a atuação fonoaudiológica tem se ampliado e de que o cuidado deve se estender a toda a família.

Observou-se que, entre as dificuldades citadas pelos fonoaudiólogos participantes, destacaram-se a compreensão da família e dos próprios profissionais sobre as condutas prestadas ao paciente, reconhecimento da equipe multiprofissional apenas na área de disfagia e a efetiva prática do princípio ético de veracidade.

\section{REFERÊNCIAS}

1. Wu MP, Tsao LI, Huang SJ, Liu CY. Development of the Readiness for Home-Based Palliative Care Scale (RHBPCS) for Primary Family Caregivers. Healthcare (Basel). 2021;9(5):608. http://dx.doi.org/10.3390/ healthcare9050608. PMid:34069437.

2. Istanboulian L, Rose L, Yunusova Y, Gorospe F, Dale C. Barriers to and facilitators for use of augmentative and alternative communication and voice restorative devices in the adult intensive care unit: a scoping review protocol. Syst Rev. 2019 Dez 6;8(1):311. http://dx.doi. org/10.1186/s13643-019-1232-0. PMid:31810494.

3. Taquemori LY. Multidisciplinaridade e Interdisciplinaridade Fonoaudiologia; In: Arantes ACL, Souza ACM, Kira CL, Sera CTM, Sakurada CK, Ramos DLP, et al. Cuidado paliativo. São Paulo: CREMESP; 2008. p. 64-6.

4. Pittioni MEM. Fonoaudiologia hospitalar: uma realidade necessária [Monografia]. Londrina: Centro de Especialização em Fonoaudiologia Clínica; 2001.

5. Luchesi KF, Silveira IC. Palliative care, amyotrophic lateral sclerosis, and swallowing: a case study. CoDAS. 2018;30(5):e20170215. http:// dx.doi.org/10.1590/2317-1782/20182017215. PMid:30184005.

6. Silva MM, Santanda NGM, Santos MC, Cirilo JD, Barricas DLR, Moreira MC. Cuidados paliativos na assistência de alta complexidade em 
oncologia: percepção de enfermeiros. Esc Anna Nery. 2015;19(3):4606.

7. Moreira MJS, Guimarães MF, Lopes L, Moreti F. Contribuições da Fonoaudiologia nos cuidados paliativos e no fim da vida. CoDAS. 2020;23(4):e20190202. http://dx.doi.org/10.1590/2317-1782/20202019202.

8. Santana PPC, Silva JR, Matias TF, Silva GCA, Ribeiro WA, Andrade $\mathrm{M}$. Speech therapy practice to patients in palliative care: an integrative review. Research, Society and Development. 2020;9(8):e108985464. https://doi.org/10.33448/rsd-v9i8.5464.

9. Silva AAA, Pestana FKM, Rocha FC, Rios BRM, Aquino AA, Gonçalves Sobrinho JF, et al. Percepção de profissionais da saúde sobre eutanásia. Rev Bioet. 2020;28(1):111-8. http://dx.doi.org/10.1590/198380422020281373 .

10. Minayo MCS. O ciclo da pesquisa. In: Minayo MCS, Deslandes SF, Neto OC, Gomes R. Pesquisa Social: teoria, método e criatividade. 34. ed. Petrópolis, RJ: Vozes; 2015.

11. Bardin L. Análise de conteúdo. São Paulo: Edições 70; 2011.

12. Silva EP, Sudigursky D. Conceptions about palliative care: literature review. Acta Paul Enferm. 2008;21(3):504-8. http://dx.doi.org/10.1590/ S0103-21002008000300020.

13. Schramm FR. Morte e finitude em nossa sociedade: implicações no ensino dos cuidados paliativos. Rev Bras Cancerol. 2002;48(1):17-20. http://dx.doi.org/10.32635/2176-9745.RBC.2002v48n1.2258.

14. Silveira MH, Ciampone MHT, Gutierrez BAO. Percepção da equipe multiprofissional sobre cuidados paliativos. Rev Bras Geriatr Gerontol. 2014;17(1):7-16. http://dx.doi.org/10.1590/S1809-98232014000100002.

15. Carvalho RT, Taquemori LY. Nutrição e Hidratação; In: Carvalho RT, Parsons HA. Cuidado Paliativo. São Paulo: CREMESP; 2008. p. 236.
16. Pollens R. Role of the speech-language pathologist in palliative hospice care. J Palliat Med. 2004;7(5):694-702. http://dx.doi.org/10.1089/ jpm.2004.7.694. PMid:15588361.

17. Carro CZ, Moreti F, Pereira JMM. Proposta de atuação da Fonoaudiologia nos Cuidados Paliativos em pacientes oncológicos hospitalizados. Distúrb Comun. 2017;29(1):178-84. http://dx.doi.org/10.23925/21762724.2017v29i1p178-184.

18. Noureddine N, Hagge D, Kashkouli P. Student-reported attitudes during an interprofessional palliative care learning experience: implications for dual-professional identity, interdisciplinary bias, and patient outcomes. Palliat Med Rep. 2020;1(1):307-313. http://dx.doi. org/10.1089/pmr.2020.0096. PMid:34223490.

19. Calheiros A, Albuquerque C. A vivência da fonoaudiologia na equipe de cuidados paliativos de um Hospital Universitário do Rio de Janeiro. Revista Hospital Universitário Pedro Ernesto. 2012;11(2):94-8.

20. Lucateli MCS, Zancanella MS. Dificuldades de comunicação e deglutição em pacientes que necessitam de cuidados paliativos. Passo Fundo: Curso de Fonoaudiologia. Universidade de Passo Fundo; 2018. p. 23. Artigo de conclusão de curso.

21. Santana JCB, Wenceslau DR, Martins FS, Almeida MF, Costa MMS. Cuidados paliativos nas unidades de terapia intensiva: implicações na assistência de enfermagem. Rev Enferm. 2012;16(3):327-43.

22. Brasil. Ministério da Saúde. Conselho Nacional de Saúde. Resolução $n^{\circ} 41$, de 31 de Outubro de 2018. Dispõe sobre as diretrizes para a organização dos cuidados paliativos, à luz dos cuidados continuados integrados, no âmbito Sistema Único de Saúde (SUS). Diário Oficial da União; 2018.

23. Maciel MGS. Definições e princípios. In: Carvalho RT, Parsons HA. Cuidado paliativo. São Paulo: CREMESP; 2008. p. 15- 32. 


\section{ANEXO 1. Entrevista}

Nome:

Gênero:

Identificação profissional/ titulação acadêmica:

Atuação:

Tempo de atuação enquanto Fonoaudiólogo:

Tempo de atuação na área de Hospitalar:

Tempo de formação:

1. Você já atuou com pacientes em cuidados paliativos?

2. Qual sua percepção sobre o que é cuidados paliativos?

3. Na sua opinião, qual o papel da fonoaudiologia nos pacientes em cuidados paliativos?

4. Na sua opinião, qual você considera as diferenças entre os atendimentos realizados em cuidados paliativos e o restante de atendimentos realizados na área hospitalar?

5. Qual a sua visão sobre o trabalho na equipe multidisciplinar em cuidados paliativos?

6. Quais as dificuldades que você já encontrou na sua atuação com cuidados paliativos?

7. Você percebe se há reconhecimento dos outros profissionais perante seu trabalho como fonoaudiólogo? Por que? 